\title{
Analytical Calculation of Magnetic Flux Lines in 3-D Space
}

\author{
Vlatko Čingoski ${ }^{\dagger}$ Manabu Ichinose Kazufumi Kaneda Hideo Yamashita \\ Electric Machinery Laboratory, Faculty of Engineering \\ Hiroshima University, Kagamiyama 1-4 \\ Higashi-hiroshima, 724 JAPAN
}

\begin{abstract}
We propose a new analytical method for computation of magnetic flux lines in 3-D space. Using the results obtained from finite element analysis and the magnetic flux line equation in 3-D space, the sequence of line segments that construct the magnetic flux line is computed analytically. The proposed method reduces computational time by nearly five times for the same accuracy with existing procedures, providing cheap and efficient computation. The procedure and some examples to present the usefulness of the proposed method are described.
\end{abstract}

\section{INTRODUCTION}

Large-memory, high-speed computers together with progress in numerical analysis, have assisted researchers in tackling the solution of increasingly complex and higherdimensioned fields. With these developments, demand for good visualization techniques of computation results has been increasing [1]. For example, one common method for aiding in understanding the behavior of three-dimensional magnetic fields is the graphic illustration of magnetic flux line distribution. By displaying magnetic flux lines, the observer can clearly and simultaneously understand the direction, magnitude and loci (stream lines) of a vector field. The authors have already succeeded in stereo visualization of magnetic flux lines calculated from results of finite element analysis [2]. Magnetic flux lines correspond to stream lines in fluid flow areas, for which many experimental visualization methods have been proposed [3]. The imaginary particle tracing method [4], used in [2], is a general computational method for magnetic flux lines. Unfortunately, this method for calculating the magnetic flux lines leaves the following problems unsolved:

1. Discontinuity of the magnetic density vector on the element boundaries.

2. Cumulative error.

3. Computational cost.

To solve the aforementioned problems, in this paper the authors propose a new analytical method for the computation of magnetic flux lines, using results obtained by the $A-\phi 3-D$ finite element method with second order tetrahedral elements. To solve the first problem, we adopted

\footnotetext{
Manuscript received November 1, 1993.

†The author is no leave from Electrotechnical Faculty, University "Sv. Kiril i Metodij", Skopje, Macedonia
}

a first order tetrahedron finite element as a visualization mesh where the values of the magnetic flux density vector at each node are defined. To solve the second and third problems, we developed a new analytical procedure. The procedure and some examples are described to present the usefulness of the proposed method.

\section{Problems of Traditional Method}

As previously mentioned, the imaginary particle tracing method is a general computational method for calculating magnetic flux lines. Unfortunately, however this method does not solve the following problems:

1. Discontinuity of the magnetic density vector on the element boundaries: Because the magnetic flux density vector is obtained by rotation of magnetic vector potential, discontinuity in the magnetic flux density vector occurs on the element boundaries. An example of this is the magnetic flux density vectors at point $P$ on the boundary between the two elements $a$ and $b$ in the 2-D model shown in Fig. 1. Occasionally, the magnetic flux density vectors $\mathbf{B}_{a}$ and $\mathbf{B}_{b}$ calculated by using vector potential of the elements $a$ and $b$, respectively, turn toward the elements $b$ and $a$. This discontinuity makes calculation of the magnetic flux line at point $P$ impossible.

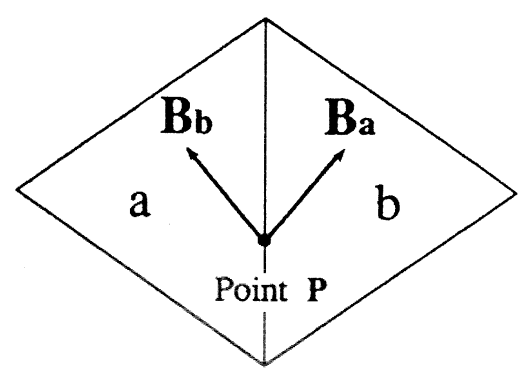

Fig. 1. Magnetic flux density vectors on element boundaries.

2. Cumulative error: The magnetic flux line equation is given as follows

$$
\frac{d x}{B_{x}}=\frac{d y}{B_{y}}=\frac{d z}{B_{z}}=d t
$$


where $t$ is auxiliary (supplemental) variable. The magnetic flux line at point $\mathbf{P}_{0}\left(x_{0}, y_{0}, z_{0}\right)$, which is traversed by the line, is approximated by a short line segment whose direction corresponds to that of the magnetic flux density vector at that point. The next point on the magnetic flux line $\mathbf{P}_{1}\left(x_{1}, y_{1}, z_{1}\right)$, is calculated by

$$
\mathbf{P}_{1}=\mathbf{B}\left(\mathbf{P}_{0}\right) \cdot \Delta s+\mathbf{P}_{0}
$$

where $\Delta s$ is a step width for iterative calculation. This procedure in each step leads to cumulative error as shown in Fig. 2.

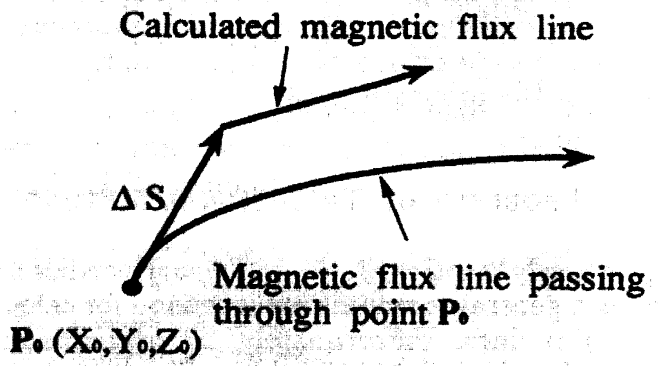

Fig. 2. Cumulative error.

3. Computational cost: To obtain highly accurate magnetic flux lines, the number of computational points by which magnetic flux lines pass must be adequate and the value of $\Delta s$ should be small. Higher accuracy, therefore, automatically indicates an increase in both time and cost for computation.

\section{OUTLNE OF THE PROPOSED METHOD}

To solve the first and second aforementioned problems, as well as to realize high speed display, various measures are taken: First a 3-D display space, which is taken from the analyzed region or a part thereof, is divided into a regular mesh of hexahedra (see Fig. 3). Next, as a new visualization mesh, a hexahedron by which the magnetic flux line under calculation closely passes is divided into six tetrahedra. This new visualization mesh in general is not the same with computation mesh. The values for magnetic flux density vector B at each node of the visualization mesh are calculated via finite element analysis. By adopting the visualization mesh, the second and third problems are easily solved and the magnetic flux line in each tetrahedron can be analytically calculated very quickly.

The three components $B x, B y$, and $B z$ of the magnetic flux density vector at the arbitrary point $P(x, y, z)$ inside a tetrahedron are given as follows:

$$
\left\{\begin{array}{l}
B_{x} \\
B_{y} \\
B_{z}
\end{array}\right\}=\left[\begin{array}{lll}
a_{1} & b_{1} & c_{1} \\
a_{2} & b_{2} & c_{2} \\
a_{3} & b_{3} & c_{3}
\end{array}\right] \cdot\left\{\begin{array}{l}
x \\
y \\
z
\end{array}\right\}+\left\{\begin{array}{l}
d_{1} \\
d_{2} \\
d_{3}
\end{array}\right\}
$$

where $a_{k}, b_{k}, c_{k}, d_{k},(k=1,2,3)$ are constants determined by the nodal coordinates $x_{j}, y_{j}, z_{j}$ and the values of magnetic flux density vectors $\mathbf{B}_{j},(j=1, \ldots, 4)$ at the four

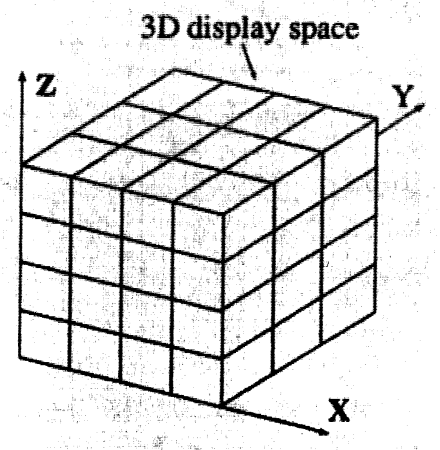

Fig. 3. Display space.

nodes of a tetrahedron. By substituting (3) into (1), we get

$$
\frac{d x}{d t}=\mathbf{E x}+\mathbf{f}
$$

where

$$
\begin{array}{r}
x=(x, y, z)^{T}, \\
\mathbf{E}=\left[\begin{array}{lll}
a_{1} & b_{1} & c_{1} \\
a_{2} & b_{2} & c_{2} \\
a_{3} & b_{3} & c_{3}
\end{array}\right], \\
f=\left(d_{1}, d_{2}, d_{3}\right)^{T},
\end{array}
$$

and $T$ stands for transpose. Applying the Laplace transformation to (4) leads to

$$
\mathbf{x}(t)=e^{\mathbf{E} t}\left(\mathbf{x}_{\mathbf{0}}+\mathbf{E}^{-1} \mathbf{f}\right)-\mathbf{E}^{-1} \mathbf{f}
$$

where $x_{0}=\left(x_{0}, y_{0}, z_{0}\right)^{T}$ is the starting point of the flux line. For an arbitrary value of $t$ in (6), we can accurately obtain the points traversed by the magnetic flux line.

In the visualization tetrahedron mesh shown in Fig. 4, the magnetic flux line starting at point $P_{0}$ passes through point $Q$, which, at the same time, is the starting point in the adjacent finite element. It is necessary, therefore, to use the linear convergence method to accurately obtain the coordinates of point $Q$. By using the linear convergence method for $t$, the volume coordinate $\xi_{i}$, is made zero and we can then obtain a point sufficiently close to $Q$. To reduce the number of iterations in the linear convergence method, the estimated value $t$ at point $Q$ is obtained by the following method. As shown in Fig. 4, the half-line through point $P_{0}$, the direction of which coincides with the magnetic flux density vector $B_{p_{0}}$ at point $P_{0}$ is considered. First, we calculate the points of intersection $Q_{j}^{\prime}$ on the half-line and the three planes including the triangular planes of the tetrahedron element except the plane that contains point $P_{0}$, where $j$ is equal to or less than 3 . Then, point $Q_{j}^{\prime}$ is selected where $\overline{P_{0} Q_{j}^{\prime}}$ is shortest. The value of $t$ at point $Q$ is estimated from the length of segment $\overline{P_{0} Q_{j}^{\prime}}$. Point $Q$ on the surface of the tetrahedron is calculated by making one of four volume coordinates, $\xi_{j}$, converge towards zero. If all volume coordinates at point $Q, \xi_{j},(j=1, \ldots, 4)$ satisfy the condition $0 \leq \xi_{j} \leq 1$, the 


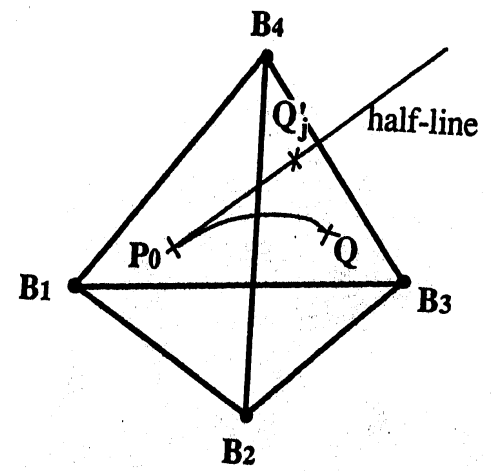

Fig. 4. Estimation of $t$ between points $P_{0}$ and $Q$.

magnetic flux line exits the element through that point. If $\xi_{j}$ is either negative or greater than 1 , the second shortest
length of $\overline{P_{0} Q_{j}^{\prime}}$. is adopted for obtaining $Q$

\section{Magnetic Flux line Calculation AlgorithM}

First, the observer sets a 3-D display space in which he whape of this space is rectangr of magnetic flux lines. The display space is dividectangular as shown in Fig. 3. The by arbitrary subdivision into a regular mesh of hexahedra that is, $x, y, z$. Values for the for all three directions, tor $B$ at each node of the magnetic flux density vecfinite element analysis and hexahedra are calculated via ory. For existing material put into the computer's memthe display space, ma menticth different permeabilities in tinuously on the, magnetic flux density changes disconboundary, therefore, are boundary. The nodes on the of magnetic flux de, are duplicated, and the calculation

The algorithm for passing through the arbitrary the magnetic flux line below. The magnetic flux lin starting point $P_{0}$ is shown of points, where the distance is displayed as a sequence sequence is controlled by the between two points in the

arbitrary basic distance $\Delta s$. ing the starting the rectangular element $\mathcal{R}_{r}$, includeight grid nodes, as shown in $P_{0}$, where constructed by

Step 2: Divide the rectangular element $\mathcal{R}_{r}$ into six tetrahedron elements, $T_{1} \sim T_{6}$, as shown in Fig. 5(b).

- Step 3: Extract the tetrahedron element $T_{0}$, including $P_{0}$, from the set of tetrahedra $T_{1} \sim T_{6}$.

- Step 4: Calculate point $Q$, where the magnetic flux in the previous section.

- Step 5: If $\overline{P_{0} Q}$ is greater than $\Delta s$, the sequence of points which are generated by dividing the magnetic flux line between $P_{0}$ and $Q$ into $n$ equal parts are calculated from (6), where $n$ is calculated by

$$
n=\left[\frac{\overline{P_{0} Q}}{\Delta s}\right]+1
$$

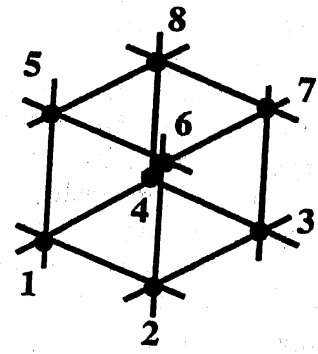

a) Rectangular region $\mathcal{R}_{r}$

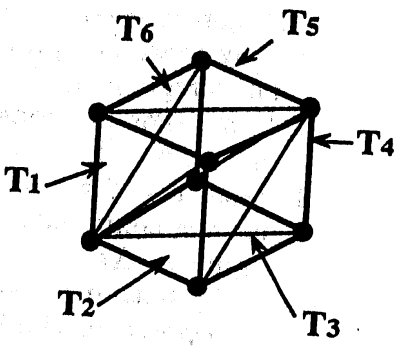

b) Division map where [ ] shows truncation for integer.

- Step 6: Point $Q$ is then re-set as point $P_{0}$. If the tetrahedral element excluding $T_{0}$ and including $P_{0}$ exists in $\mathcal{R}_{r}$, the tetrahedron is set as $T_{0}$ and go to the next step.

- Step 7: If point $P_{0}$ reaches the boundary of the display space, the process is finished. Otherwise, the adjacent rectangular element with $\mathcal{R}_{r}$ and including $P_{0}$ is renamed as $\mathcal{R}_{r}$, and go to Step 2 .

By using the algorithm mentioned above, the magnetic flux line in the positive direction of the magnetic flux density vector at the starting point $P_{0}$ can be calculated. The magnetic flux line that lies in the opposite direction from point $P_{0}$ is calculated by selecting the opposite direction the following charax density vector. This algorithm has ements is sufficient onlytics: Generating tetrahedral elwhen calculating magnetic the rectangular element $\mathcal{R}_{r}$ sary to keep in magnetic flux density; it is not necesdisplayed space. As the grid tetrahedral elements in the orizing the coordina grid nodes line up regularly, memadvantageous from the point af the nodes is unnecessary, itations. In addition, it is easy view of memory-size limelement $\mathcal{R}_{\boldsymbol{r}}$ through, it is easy to select the rectangular also advantageous for keeping magnetic flux line passes, imum.

\section{Results and ApPLICATIONS}

The main feature of the proposed method is its low computation cost for such high-accuracy display. To obtain sufficiently accurate magnetic flux lines in [2], we must reduce the length of the line segment $\Delta s$ as much as posproposed methoder, lengthens computation time. In the computed by giving the magnetic flux line is analytically arbitrary value of $t$, and as the point of the line and an ular, finding the $t$, and as the visualization mesh is regline passes is easy, decreasing in which the magnetic flux demonstrate the proposed metho computation time. To of a rectangular core surrounded, a model constructed two aluminum plates was analyzed a rectangular coil and metrically set above and analyzed. The plates are sym- 
method was then compared with the traditional method [2]. The display space $250[\mathrm{~mm}] \times 200[\mathrm{~mm}] \times 200[\mathrm{~mm}]$, was divided into regular mesh of hexahedra with constant width of $5[\mathrm{~mm}]$. For one magnetic flux line, the results are shown in Fig. 6. The computation time is presented in Table I. From Fig. 6 we can see that using method [2] for longer line segments enlarges the computation error. Using line segments $\Delta s=1 \cdot 10^{-6}[\mathrm{~m}]$, the magnetic flux lines obtained by both methods have almost the same accuracy. The proposed method, however, reduced computation about time by nearly five times. In Fig. 7 the distribution of the magnetic flux lines in the entire analyzed domain is presented. Due to the existence of eddy current distribution in the aluminum plates, magnetic flux lines are obstructed near the aluminum plates. Fig. 8 shows the eddy current stream lines. The observer can easily see that the eddy current stream lines are warped near the longer edge of the aluminum plates. The computation was performed on a Silicon Graphics IRIS - 4D / 20G CPU (10 MIPS) computer.

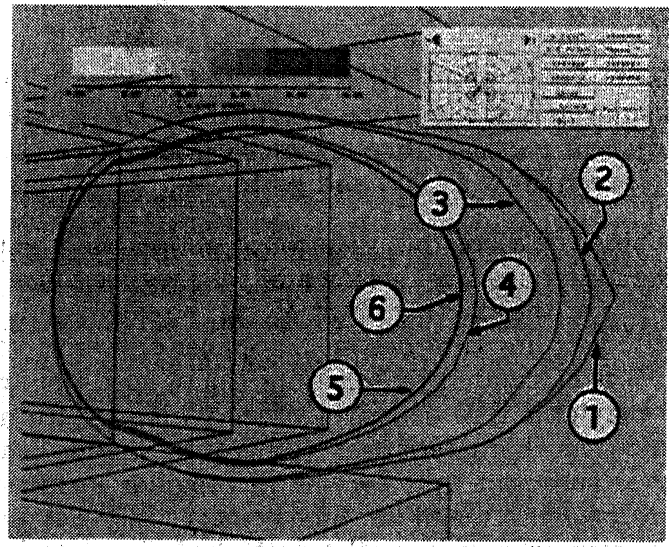

Fig. 6. Comparison between traditional and proposed method.

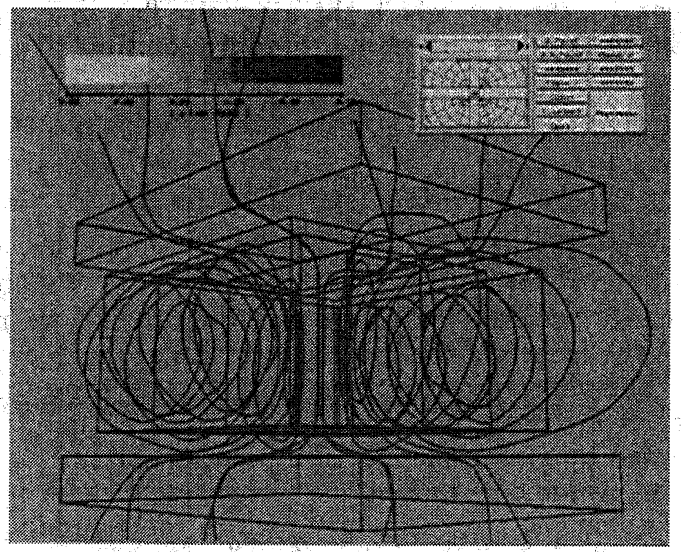

Fig. 7. Visualization of magnetic flux lines.

\section{CONCLUSIONS}

A new analytical method for computation of magnetic flux lines with higher accuracy and reduced computation

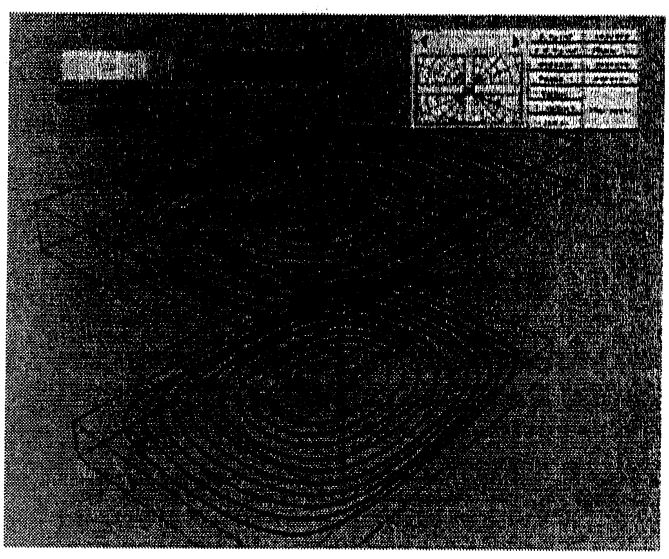

Fig. 8. Visualization of eddy current lines.

TABLE I

Results comparison

\begin{tabular}{|c||c|c|c|c|c||c|}
\hline \multicolumn{1}{|c||}{} & \multicolumn{5}{c||}{ Traditional Method } & $\begin{array}{c}\text { Proposed } \\
\text { Method }\end{array}$ \\
\hline \hline$\Delta \mathrm{s}(\mathrm{m})$ & $10^{-2}$ & $10^{-3}$ & $10^{-4}$ & $10^{-5}$ & $10^{-6}$ & $10^{-2}$ \\
\hline$t(\mathrm{sec})$ & 3 & 3 & 5 & 5 & 10 & 2 \\
\hline line & 1 & 2 & 3 & 4 & 5 & 6 \\
\hline
\end{tabular}

time was presented. The main features of this method are as follows:

1. Continuity of magnetic flux lines is satisfied with decreased computation time and cost.

2. Adoption of the visualization mesh ensures continuity of the magnetic flux density vector at element boundaries.

3. The proposed method can be applied to other analytically or numerically obtained yector data, such as BEM.

The problems which arise in visualization of the rapidly varying fields by uniform grid, can be overcome by subdivision of the display space into a few sub-display areas where again regular but differently dense grids can be used. The analytically obtained ending points from one regular grid, can be used as a starting points of the same magnetic flux line in the adjacent regular grid.

\section{REFERENCES}

[1] McCormick, B. H., deFanti, T. A., and Brown, D. B. eds., "Visualization in Science Computing",Computer Graphics, 21, 6, (Nov. 1987)

[2] Yamashita, H., Harada, K., Nakamae, E., Itano, J., and Hammam, M. S. A. A., "Stereo Graphic Display on Three Dimensional Magnetic Fields of Electromagnetic Machines", IEEE Trans. on Power Apparatus and Systems, PAS-100, No.6, p. 4692 (1981).

[3] Asanuma, K., Flow Visualization Handbook, Asakura, Tokyo, 1977 (in Japanese).

[4] Shiroyama, S., "Flow Visualization by Imaginary Particle Tracing Method", The 4th symposium of Numerical methods in flow dynamics, p.483 (1990) (in Japanese). 